\title{
Cloud Computing Solution and Services for RFID Based Supply Chain Management
}

\author{
S. M. Khalid Jamal ${ }^{*}$, A. Omer ${ }^{1}$, Asiya Abdus Salam Qureshi ${ }^{2}$ \\ ${ }^{1}$ Department of Computer Science, UBIT, University of Karachi, Karachi City, Pakistan \\ ${ }^{2}$ Department of Computer Science and Software Engineering, University of Hail, Hail, KSA \\ Email: *s.m.khalid@uok.edu.pk
}

Received July 31, 2013; revised September 5, 2013; accepted September 23, 2013

Copyright (C) 2013 S. M. Khalid Jamal et al. This is an open access article distributed under the Creative Commons Attribution License, which permits unrestricted use, distribution, and reproduction in any medium, provided the original work is properly cited.

\begin{abstract}
Deploying and Implementing RFID Systems for tracking and controlling products in supply chain management is not affordable for small and midsized companies due to its complexity, cost, and difficulties pertaining data management and maintenance. Cloud computing can be used to mitigate the risks associated with the implementation and deployment of RFID based on system in supply chain management because the complex system for data filtering, management and maintenance can be implemented on the cloud that otherwise requires the purchase of new hardware, staff and professional services.
\end{abstract}

Keywords: Cloud Computing; RFID; Tags; Readers; Barcodes

\section{Introduction}

Radio Frequency Identification (RFID) is a prevailing technology that uses radio waves to monitor and control the products in supply chain management. Each product has a tag affixed to it during manufacturing process which contains a unique identifier that can be used to uniquely identify a product by all supply chain participants. The deployment as well as implementation of RFID systems has been a challenging task for small and medium sized enterprises (SMEs). In this paper, we propose cloud computing solution to overcome these challenges. The supply chains can install RFID solution much faster by using the cloud implementation while avoiding many of the expensive infrastructure cost required for pre-cloud systems.

RFID technology has been introduced across a range of industries, including public sector as well as inretail and manufacturing. For example, RFID has been used by Harley-Davidson and Toyota to monitor parts for their motorcycles and automobiles, respectively. Furthermore, baggage at Las Vegas Airport is tagged to enhance and improve loading procedures as well as the tracking of passengers' baggage. The US Department of Defense and Wal-Mart hosted RFID technology a decade ago, and required their suppliers to make all supplies RFID en-

${ }^{*}$ Corresponding author. abled, in order to ease its inventory management. For organizations of all types, the ability to quickly locate and enumerate products may facilitate business value of supply chain management [1].

Small and midsized companies are unable to afford the costs and risks associated with the implementation and deployment of RFID systems. Therefore, we propose a cloud computing solution so that the capital investment and the return-on-investment risks associated with the new technology of RFID can be mitigated. Cloud-base services are designed in such a way as to eliminate the hardware and software infrastructure requirements whose implementation is time consuming as well as expensive. Moreover, it provides abstraction to its users by hiding all the complex details of the system. Users can only use the provided services by interacting with application software responsible for receiving inputs and providing outputs. The inner workings are completely invisible [2].

\subsection{Motivation}

According to an estimate, the annual savings of US leading retail Wal-Mart using RFID are $\$ 8.35$ billion. This huge amount is divided as follows: it saves $\$ 600$ million by meeting timely delivery of goods due to real-time and rapid information that RFID systems provide; inventory shrinkage saves $\$ 180$ million; $\$ 575$ million are saved by controlled tracking and monitoring of the products that 
prevents theft, mistakes and buyer fraud; pallets and the need to scan each product individually which reduces the labor cost as well as time. By implementing RFID, the total of \$3 billion annual investment is made by WalMart as opposed to $\$ 8.35$ billion made earlier. The annual savings in different heads are represented in the Bar-

\section{Graph 1.}

Due to its speed, accuracy and improved inventory management, RFID technology has been widely adopted and recognized as an emerging and affective technology in industry and businesses. An RFID system is made of up to following three arrangements:

- An RF subsystem, which uses wireless communication of RFID tags and readers to perform wirelessidentification of tagged items over a network [3].

- An enterprise subsystem, that stores, processes and manages the data received from the RF subsystem through software applications designed for this purpose and that makes the RFID data more powerful.

- An inter-enterprise subsystem, through which multiple enterprise subsystems are connected in order to share information among supply chain networks [4]. All RFID systems have an RF subsystem where the tags are read in and almost all RFID systems also include some form of an enterprise subsystem. An inter-enterprise subsystem is used when the information needs to be shared across the enterprise boundaries.

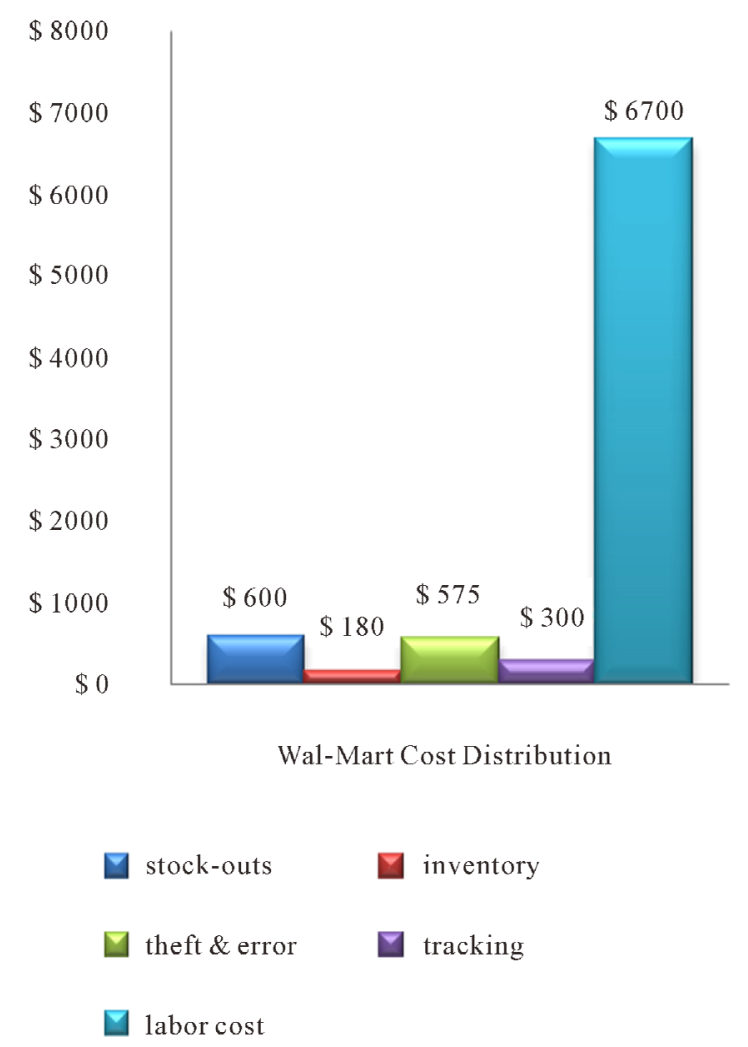

Graph 1. Annual savings by Wal-Mart in different heads.
The RF subsystem is composed of two basic components in order to identify the tagged items.

- RFID tags also called transponders are small electronic chips that are either attached to items or embedded within them. All tags can be uniquely identified by an identifier assigned to them. These devices may have their own storage space containing additional information about the tagged item and sensors attached to them to monitor temperature for example or other environmental and status conditions.

- RFID readers are devices that are used to retrieve the information stored on RFID tags and identify each tag uniquely by wirelessly communicating with the tags [5].

Tags can be active, having their own source for power supply or passive, depending upon the RFID reader to supply the power necessary to transmit signals. The signal can be a simple identification number, or a complex data stream stored in the tag's memory. These tags can contain information such as the expiry date, date of manufacturing, built in sensors to track temperature or other data [6].

\subsection{RFID in Supply Chain Management}

The most data rich and intensive RFID application is the supply chain management systems in which a tagged product is tracked from manufacture to final purchase, throughout its life cycle. In addition to process data, data information is also maintained in these systems, such as the formats in which it is stored and transmitted by the supply chain in various organizations and the database network addresses that contains data about different tagged items.

Supply chain management is also used in monitoring and product control from manufacture to retail sail including its distribution. It is typically a collection of different kind of application types, which includes asset management, process control and tracking and payment systems.

In supply chain systems, the information about the product can be stored at every phase in the supply chain. Tags are attached to products during manufacturing process or soon after it to these products in ideal situation. As the product progresses through the supply chain, the information on these tags can be altered by the supply chain participants. Moreover, larger objects such as pallets and containers can be tracked and monitored by these systems using active tags. These tags which are affixed on the pallets can store information about the items shipped in each of the container. When the products are moved out from the container the information which the tags carry are automatically upgraded to reflect the new information. An electronic history is also generated for each item in the container which ensures the buyers about the item's freshness, enabling them to identify 
whether it's useful, has expired or not [7].

Objects are automatically identified with the help of fully utilized EPC data. It can also be used in monitoring location and movement, and trigger events. Operational efficiencies could be gained by a near real-time view throughout the supply chain, such as improved inventory control, increasing throughput and lowering cost of the products [8].

\subsection{Benefits of Using RFID}

- Time spent in ordering products is minimized.

- Less number of employees required for inventory control.

- Manual entries in the inventory document might become prone to errors, whereas using RFID technology the entries are automatic and hence more accurate.

- Sales reports are created automatically.

- Overstocking in warehouses is minimized.

- Forecasting the demand for products can be performed more accurately because real time data is used for estimation of demand.

- Improved communication between suppliers and customers.

- Reduction in costs for labor needed for database management in inventory.

\subsection{Electronic Product Code (EPC)}

The Electronic Product Code (EPC) is a unique identifier stored on the tag that can be used to retrieve more information about the tagged item from a database. EPC is standardized by EPCglobal (a non-profit organization) and acts as a replacement to UPC (Universal Product Code) numbers when barcodes are used as identifiers on products. However, unlike UPC barcodes that refer to a class of products, EPC refer to a specific instance of product. A unique EPC serial code that is saved in the tag is transferred back to the reader when an EPC tag is read by RFID reader. The product is identified through internet using the facility of Object Naming Service (ONS). The ONS matches the specific EPC code to the corresponding entry on the internet that provides additional information about the product. EPC Information Services (EPCIS) are then used to communicate available information in real time via EPCglobal Network. The real time data that is exchanged on EPCglobal Network allows for the tracking and monitoring of products. Unlike the identification of classes of products as with barcodes, EPC allows us to uniquely identify an instance of a tagged product. A serial number embedded within the EPC is used to uniquely identify a tagged product. The set of EPCs within the domain of a person is referred to as his "EPC cloud". This EPC cloud can be used to monitor thefts within the supply chain [9].

\section{Challenges Faced in RFID Implementation}

One of the major challenges in RFID implementation is the high cost associated with its implementation. This includes the cost of installation of the new hardware and software as well as the hiring of new staff for professional services required for the integration of the new system. According to an estimate, an RFID-enabled warehouse would cost in excess of \$2 million which makes it unsuitable, especially for Small and Medium sized Enterprises (SMEs), to implement a fully-fledged RFID system.

Another challenge is the lack of understanding of RFID technology and the approaches required to integrate the RFID technology into the current IT infrastructure. Capturing, exchanging and managing data from RFID readers and integrating the data into EPCIS and sharing data among enterprises can become a challenging task. This makes the deployment and maintenance of RFID systems a time consuming and complex operation. According to a survey, two-thirds of all respondents said they are still learning about the technology; while 26.3 percent said they have an intermediate level of knowledge. The automotive industry recorded a slightly higher level of intermediate knowledge, at 33 percent, while the transportation market, at 28 percent, was slightly higher than the average for all industries. Therefore, the low awareness and lack of understanding of RFID systems among stakeholders is a critical factor preventing its adoption [10].

Another problem in RFID implementation is the heavy volumes of data that it generates that are hard to handle. According to an estimate, Wal-Mart produces 7 terabytes of operational RFID data per day. Moreover, supply chain monitoring and real time tracking requires storing additional data that the traditionally designed databases cannot manage. This implies additional hardware costs needed to manage the large volumes of data generated by RFID systems. According to an estimate, RFID based solutions and services are expected to be adopted over the next 18 - 24 months due to the prevailing acceptance of cloud computing [11].

\section{Proposed Cloud Computing Implementation}

Cloud computing is a prevailing technology in which computing infrastructure, hardware, software and services are provided wherever and whenever needed. With the proposed cloud computing architecture shown in Figure 1, data from the scanning system is provided to the EPC Information System that is implemented on cloud as SaaS (Software as a Service). The transmitted data is stored and managed on the cloud and is made 


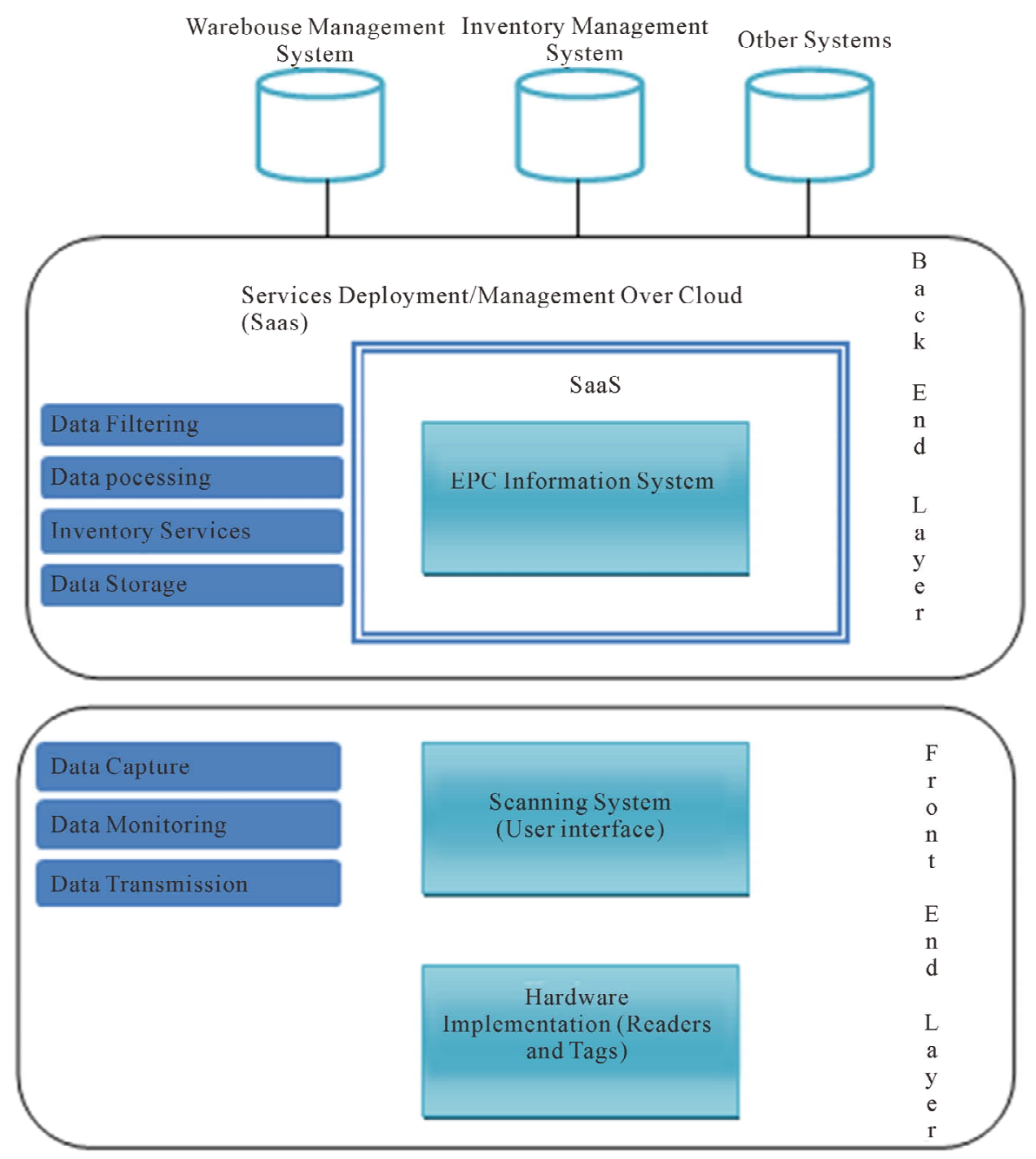

Figure 1. Cloud-based architecture.

available in a reliable manner to any application that requests it. The proposed cloud computing architecture sufficiently reduces the maintenance and support cost, which is one of the implementation barriers, while increasing overall system consistency and flexibility. Supply chains can now deploy RFID much faster while reducing the time consumption and complexity of the newly deployed system. Furthermore, the EPC Information System can also be used to implement additional functionality such as data warehousing.

\subsection{Front-End Layer}

The front-end portion of the proposed architecture includes the hardware implementation of RFID tags and readers over a network, the computers with sufficient capabilities for data storage and management as well as the front end software application that links the produced data with the cloud computing system. The front end begins to work when RFID tags and reader communicate over a network as a result of which RFID data is gener- ated. Whenever an RFID tag appears within the range of RFID reader, RFID data is produced. The data that is acquired by the RFID reader is then sent to the front-end software application containing the user interface and application program connecting the front-end and the back-end layers in order to gain access to more services and information that are stored and processed on the Cloud. Since, RFID systems generate huge amount of new data specially from sensor based active tags sending data through cellular network, this data needs to filtered, stored and processed before being used. A Cloud computing backend-end layer is used in order to achieve all of these objectives that is connected to front-end layer over a network. The cloud helps supply chains to manage heavy volumes of data. In addition, there are numerous advantages achieved by supply chain operators and customers which include the reduction in IT cost, removal of the costly infrastructure necessities, elimination of the complex technology, professional services and software updates required leading to faster path to return on in- 
vestment.

All the read EPCs are fed into the scanning system, that will search for those EPCs in Object Naming Service (ONS) and acquires the required information through EPCIS (EPC Information System) implemented on Cloud. The scanning system may be installed for example at a retail store's exit to scan all the products the customer purchases and the products that customer already had with him before purchase of new products. Since, EPCs are affixed within the product they need not be scanned individually as with barcodes and as EPC network stores information about individual products, these EPCs depict consumer habits and contribute to the business value. The cloud-based architecture allows the IT staff to concentrate more on other projects rather than dedicating a significant amount of time on RFID deployment and management.

\subsection{Enterprise Sub-System}

The enterprise subsystem provides a communication pathway for RFID readers and the computers that are running the front end application. The data obtained from RF subsystem is stored, processed and managed on these computers. For example, a retail garments store may have RFID system installed in which a unique identifier is read through RFID readers that uniquely identifies the product. The enterprise subsystem acts as a middleware that matches the identifier to the product's record stored in the database. The database records information about the product such as its price, the brand name or the manufacturing date. The proposed architecture consists of two layers the front-end layer composed of the scanning system and the hardware implementation of tags and readers, and the back-end layer containing EPCIS over Cloud [12].

\subsection{Back-End Layer}

The proposed cloud computing architecture (presented in Figure 1) contains back end layer which includes EPC Information System, data storage systems, computers and servers. The Cloud filters the data from RFID depending upon a particular application of interest. This filtered data uses the required SaaS services on the Cloud and is then stored on a specific server. The cloud computing system includes the relevant protocols and middleware that allow the networked resources, including RFID tags and readers, to communicate with each other.

The system traffic and system is managed by a server. Since RFID systems produce a huge amount of data, a lot of storage space is needed in the cloud computing system to store all the relevant information. Therefore, a copy of all RFID information is stored on the Cloud computing system. These copies are stored on backup machines and the backup data can be retrieved by the central server thus providing backup and restoration facility that was not otherwise available on traditional systems. The huge amount of data which was lost earlier at initial stages because of bearing low priority due to lack of storage space and available hardware may now be preserved and managed on the cloud to depict more intense business decisions as a result of using cloud computing. Moreover, the existing resources can be more efficiently utilized instead of demanding a need for new services and infrastructure deployment. Cloud based RFID system provides real-time data and powerful software tools. Typically the cloud offers its users additional functionalities such as warehouse and inventory management depending upon the needs of an enterprise where the client pays only for the services that are of critical importance.

\subsection{Potential in the Desired Work}

According to a study conducted at the end of 2012, 15,000 IT professionals have been approximated to use cloud based services and cloud computing which is estimated to increase rapidly in 2013. 61\% of those queried were reported to use some form of cloud computing, whereas 39\% of the respondents were not using any form of cloud services. The statistics are presented in a PieChart, i.e. Graph 2.

Furthermore it is estimated that the companies spending in cloud computing will rise to $\$ 241$ billion by 2020 from currently $\$ 40.7$ billion. Recognizing the benefits of Software as a Service and Infrastructure as a Service, $41 \%$ of small businesses plan to invest in cloud by the end of 2014 relieving the company of the need for costly capital investment and maintenance of software thus saving the costs by up to 90\%. IBM's Chief Technology Officer reports the decrease in labor cost by $50 \%$ when cloud services are used. 58\% of the companies report that cloud has offered them an improved control of data. Figure 2 shows the analysis conducted by Cisco Internet Business Solutions Group (IBSG) of the Small and Medium Businesses (SMB) total expenditure that is adoptable by cloud services (shown on the left hand side). The

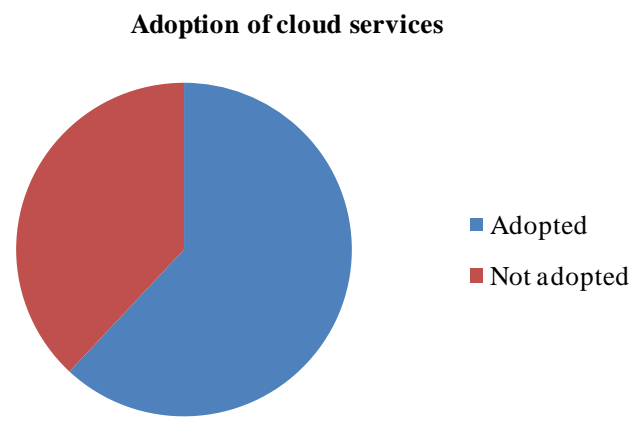

Graph 2. Pie-chart representing cloud adoption. 


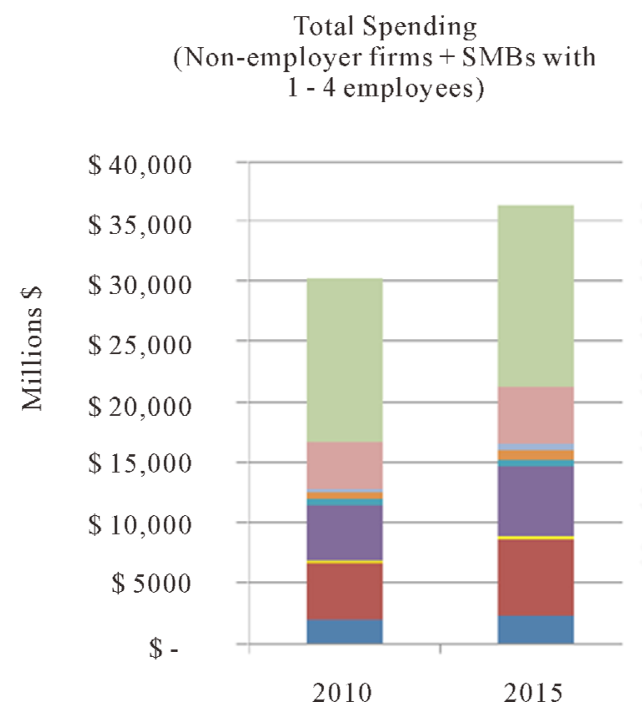

2010

2015

\section{$\$ 40,000$
$\$ 35,000$
$\$ 30,000$
$\$ 25,000$
$\$ 20,000$
$\$ 15,000$
$\$ 10,000$
$\$ 5000$
$\$-$}

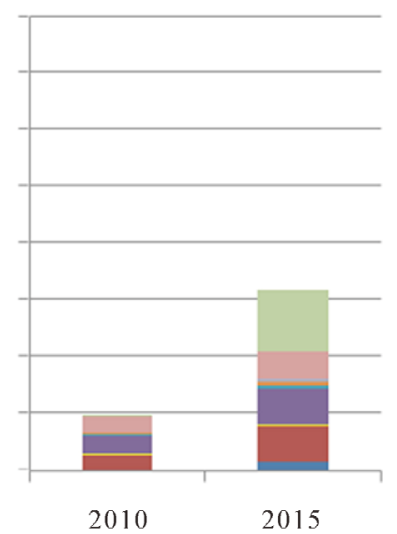

Cloud Spending

(Non-employer firms + SMBs with

1 - 4 employees)

Source: Cisco IBSG. 2012

Figure 2. Between 2010 and 2015, US SMB cloud spending will increase from $16 \%$ of cloudable spending to $44 \%$, or $\$ 16$ billion, driven by tax and accounting applications and web hosting.

right hand side of the figure depicts the rates when cloud services are adapted to the spending on the left [13]. The query regarding what proportion of today's spending in IT services will ultimately move to a public cloud is unanswerable since the cloud offers on demand and flexible expenditure in contrast to the fixed contract in usual business scenario. Cisco IBSG research also depicts the high consideration of SMB towards the migration to cloud based services [14].

Analysis by Cisco IBSG shows that the total SMB expenditure on cloud adoptable services will rise from $\$ 31$ billion in 2010 to \$36 billion in 2015 whereas the expenditure on actual cloud based services will rise from $\$ 4$ billion to $\$ 16$ billion within the same period of time [15].

\subsection{Benefits of Cloud Based Architecture}

- One of the biggest challenges faced in RFID deployment is its associated cost. The proposed cloud computing solution provides its clients the facility to use on-demand and pay as you go services. Thus, clients will be able use RFID technology without having to make a huge investment on the fully fledged infrastructure. Small and Medium sized businesses that cannot afford the cost of deployment and implementation of new technology can benefit from cloud computing solution [16].

- The clients need not invest in the hiring of new staff or training of the current staff for the operation and maintenance of the new complex system as it will be provided as a service on cloud.

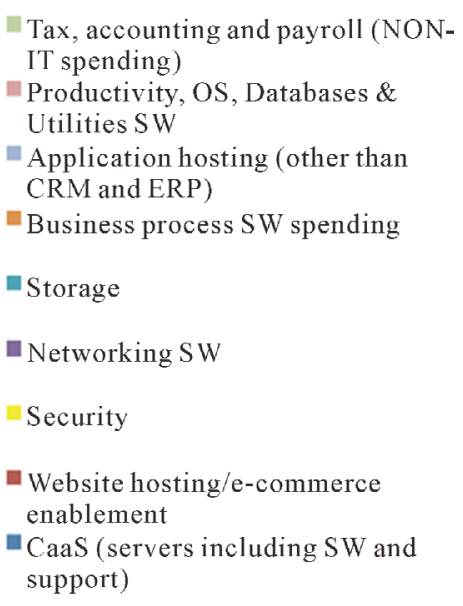

- Since, RFID produces large volumes of data that is difficult to store and manage in traditional database systems, this large volume of data can be stored and managed on the cloud on a specific server and is filtered appropriately.

- The copies of RFID data are stored on backup machines and the backup data can be retrieved by the central server thus providing backup and restoration facility that was not otherwise available on traditional systems [17].

- The vast amount of data which was lost previously at initial stages because of bearing low priority or due to lack of storage space and available hardware may now be preserved and managed on the cloud to depict more intense business decisions as a result of using cloud computing.

- Furthermore, cloud computing provides the facility of elastic capacity which means that the storage capacity and services provided are always flexible. More server resources can be allotted as and when the demand increases. When the demand decreases, there is no need to pay for the unused resources or storage space. This way the companies that are newly adopting the technology can initially demand less resources, thereby reducing the risk of return on investment on the RFID system. Then depending on the ROI, either more resources are acquired or the allotted resources are returned if the project fails [18].

- Cloud based RFID architecture is of importance to supply chains where condition based information is of 
central importance as these systems provide real time, rapid and meaningful information sharing [19].

\section{Conclusion}

This paper discusses how cloud-based solutions can be coupled together with RFID-based sensing and monitoring systems to provide on-demand information to organizations. Supply chains can more efficiently and accurately trace products and monitor condition-based information such as expiration dates, temperature and delivery status thereby allowing the stakeholders to optimize their supply chains and use real-time information to make business decisions. The cloud computing solution can benefit small and medium sized enterprises (SMEs) because they can use RFID technology without making a major financial investment on new hardware and software purchase needed for data storage and management. Since lack of understanding is one of the biggest hurdles in RFID implementation, the proposed solution relieves its users from the complex infrastructure requirement. Cloud computing solution for RFID-based systems bears the ability to revolutionize the supply chains by cutting IT costs, overstocking and efficiently manage consumer goods.

\section{REFERENCES}

[1] GSA Smart Card Website. http://www.smart.gov/

[2] K. Finkenzeller, "RFID Handbook: Fundamentals and Applications in Contactless Smart Cards and Identification,” 2nd Edition, John Wiley \& Sons Ltd., Munich, 2003.

[3] Automatic Identification Technology Office. http://www.dodait.com/

[4] L. Sullivan, "RFID Implementation Challenges Persist, All This Time Later,” Information Week, 2005. http://www.informationweek.com/rfid-implementation-ch allenges-persist-a/171203904?pgno $=1$
[5] RFID Journal. http://www.rfidjournal.com/

[6] T. Hey, S. Tansley and K. Tolle, "The Fourth Paradigm: Data-Intensive Scientific Discovery, Microsoft Research,” Microsoft Corporation, 2009.

[7] Auto-ID Labs. http://www.autoidlabs.org/

[8] FCC OET Bulletins. http://www.fcc.gov/oet/info/documents/bulletins/

[9] EPC Global. http://www.epcglobalinc.org/

[10] NIST Computer Security Guideline Publications. http://csrc.nist.gov/publications/

[11] "Cisco Global Cloud Index: Forecast and Methodology, 2011-2016,” Cisco, Whitepaper, 2012.

[12] D. Guinard, M. Mueller and J. Pasquier, "Giving RFID a REST: Building a Web-Enabled EPCIS,” Proceedings of the Internet of Things 2010 International Conference, Tokyo, November 2010.

[13] “New Supply Chain Dynamic: Providing Actionable Data through Cloud-Based Services and Solutions," RFID Journals, Whitepaper, February 2013.

[14] D. P. Handler, J. Barbier and P. Schottmiller, "SMB Public Cloud Adoption: Opening a Hidden Market,” Cisco Internet Business Solutions Group (IBSG), 2012.

[15] International Organization for Standardization. http://www.iso.org/

[16] S. Zhang, S. Zhang, X. Chen and X. Huo, "Cloud Computing Research and Development Trend,” Proceedings of the International Conference on Future Networks, 2010.

[17] D. Guinard, C. Floerkemeier and S. Sarma, "Cloud Computing, REST and Mashups to Simplify RFID Application Development and Deployment," Proceedings of the Second International Workshop on Web of Things, New York, 2011. http://dx.doi.org/10.1145/1993966.1993979

[18] A. A. S. Qureshi and S. M. K. Jamal, "Taxonomy Based Data Marts,” International Journal of Computer Application, 2012.

[19] A. A. S. Qureshi and S. M. K. Jamal, "Web Supported Query Taxonomy Classifier," International Journal of Computer Application, 2012. 